\title{
Survey of Her2-neu Expression and its Correlation with Histology of Gastric Carcinoma and Gastroesophageal Junction Adenocarcinoma
}

\author{
Seyed-Hamid Madani ${ }^{1}$,Ali Rahmati ${ }^{1}$, Edris Sadeghi ${ }^{2}$, Sedighe Khazaei ${ }^{1}$, Masoud \\ Sadeghi $^{3}$, Mehrdad Payandeh ${ }^{3}$, Nasrin Amirifard ${ }^{2 *}$
}

\begin{abstract}
Background: There is increasing evidence that HER2-neu is an important biomarker in gastric carcinomas (GC) and gastroesophageal junction (GEJ) adenocarcinomas. The aim of this study was to evaluate HER2-neu expression and also some clinicopathological features of these neoplasms. Materials and Methods: We selected 211 paraffin-embedded blocks, 193 GC and 18 GEJ. Then 4 micron sections were prepared for staining with hematoxylin and eosin and also for IHC (Her2-neu). The Chi-square test was used for significance between expression of HER2-neu and clinicopathological parameters. Results: In patients with advanced cancer of GC and GEJ, HER2-neu overexpression was more associated with the intestinal cancer subtype. Conclusions: This could be a guide to new complementary therapy for affected patients.
\end{abstract}

Keywords: Gastric cancer - gastroesophageal cancer - Her2-neu - IHC

Asian Pac J Cancer Prev, 16 (17), 7755-7758

\section{Introduction}

The incidence of primary gastric cancer (GC) and esophageal/gastroesophageal junction (GEJ) adenocarcinomas is increasing, and these tumors are estimated to make up the third and fifth most common cause of cancer deaths worldwide, respectively (Albarello et al., 2011). When a tumor is located at the GEJ, it is often unknown whether the tumor is of esophageal or gastric origin. This group of cancers is therefore called GEJ cancers (Moelans et al., 2011). Esophageal adenocarcinoma incidence has been rapidly increasing in western countries during the past half century, especially in Caucasian males (Hongo et al., 2009). GC is thought to result from a combination of environmental factors and accumulation of specific genetic alterations, and consequently, mainly affects older patients (Moelans et al., 2011).

The HER2-neu protein (p185, HER2-neu, ErbB-2) is a $185-\mathrm{kDa}$ transmembrane tyrosine kinase (TK) receptor and a member of the epidermal growth factor receptors (EGFRs) family malignancies(Gravalos and Jimeno, 2008) Patterns are scored as immunohistochemical (IHC) 0 (no staining or staining in $<10 \%$ of tumor cells, negative), IHC 1+ (faint/barely perceptible incomplete membrane staining in $>10 \%$ of tumor cells, negative), IHC $2+$ (weak to moderate complete membrane staining in $>10 \%$ of tumor cells, equivocal), or IHC 3+ (strong complete membrane staining in $>10 \%$ (until 2007) or $>30 \%$ (2007-now) of tumor cells, positive) (Wolff et al., 2007). Recently, studies have demonstrated that a proportion of gastroesophageal adenocarcinomas overexpress HER2neu, with an average of $19 \%$ and $22 \%$ of GC and GEJ adenocarcinomas displaying overexpression, respectively (Ross and McKenna, 2001; Hofmann et al., 2008). Also, HER2-neu overexpression and/or amplification have been observed in breast, colon, bladder, ovarian, endometrial, lung, uterine cervix, head and neck (Gravalos and Jimeno, 2008). This especially applies for IHC studies where different reagents and definitions of positivity resulted in an extremely wide range of HER2-neu positivity in almost all tumor types (Reichelt et al., 2007). Amplification of HER2-neu oncogene has become an important biomarker for identifying patients who respond to HER2-neu targeted therapy using the humanized monoclonal antibody trastuzumab (Herceptin) (Rakhshani et al., 2014). Different methods for analysis (Southern blot or fluorescence in situ hybridization (FISH)) and definitions of amplification have resulted in amplification frequencies ranging from 15-100\% in adenocarcinomas (Geddert et al., 2002).

The aim of this study is evaluation of HER2-neu expression and also some of the clinicopathological features of these neoplasms in the West of Iran. 


\section{Materials and Methods}

\section{Patients}

In this descriptive and analytical study, we selected 211 adenocarcinoma cases that 193 of them were GC and 18 of them were GEJ. Histological parameters were tumor grade (well, moderately, or poorly differentiated) and growth pattern (intestinal, diffuse, mixed and mucinous).

\section{Clinical and pathologic evaluation}

First of all, we provide instruments, apparatus, materials (like Canon Powershot G6) and chemistry compounds (like Hydrogen peroxide, Entelan glue made in Germany (Merk)), kits and colors (c-erbB-2 oncoprotein A*0485, Biotin Blocking System*X0590, EnVision+Dual Link System-HRP*K4063+Liguid DAB+Substrate Chromogen System*K3468, Target Retrieval Solution, pH $9(\times 10) *$ S2367 made in Denmark and Hematoxylin made in Spain) . After that, we prepared stock buffer (mixing ethylenediaminetetraacetic acid (EDTA) (0.4gr) with tris(hydroxymethyl)aminomethane (Tris) (2gr) and distilled water (D.W) (1000 ml) and for the IHC staining, first we charged slides with poly-L-lysin glue means that the slides were incubated for an hour in the glue 10 percent ( $10 \mathrm{ml}, 90 \mathrm{ml}$ of distilled water plus glue) and then dried at room temperature overnight. In this study, the patients admitted to pathology laboratory of Imam Reza and Biston Hospitals in Kermanshah (Iran) that were diagnosed GEJ adenocarciunoma and GC for them by pathologist, a sufficient sample size was selected from any patient and the slides were stained by hematoxylin and eosin $(\mathrm{H}$ $\&$ E) method. Then 4 micron sections were prepared for staining with $\mathrm{H} \& \mathrm{E}$ and also for IHC (Her2-neu) staining.

\section{Statistical analysis}

Statistical analyses were performed using the

Table 1. The Baseline Characteristics for all Patients (n=211)

\begin{tabular}{lrrr}
\hline Variables & $\mathrm{n}(\%)$ & Mean \pm SD & Range \\
\hline Age(year) & & $63.6 \pm 12.3$ & $24-98$ \\
Sex & & & \\
$\quad$ Male & $150(71.1)$ & & \\
$\quad$ Female & $61(28.9)$ & & \\
Location of Tumor & & & \\
$\quad$ Gastric & $193(91.5)$ & & \\
$\quad$ Distal esophagus & $18(8.5)$ & & \\
Tumor Subtype & & & \\
Intestinal & $142(67.3)$ & & \\
Diffuse & $43(20.4)$ & & \\
Musinous & $16(7.6)$ & & \\
$\quad$ Mixed & $10(4.7)$ & & \\
Tumor Differentiation & & & \\
Well & $94(44.5)$ & & \\
Moderately & $63(29.9)$ & & \\
Poorly & $54(25.6)$ & & \\
Her2-neu Score & & & \\
0 & $74(35.2)$ & & \\
1 & $53(25.2)$ & \\
2 & $54(25.6)$ & \\
3 & $25(11.8)$ & \\
Missing & $5(2.4)$ & \\
\hline
\end{tabular}

SPSS statistical software package version 19 (SPSS, Chicago, IL, USA). Chi-square test was used to analyze the significance of correlation between the expression of HER2-neu and clinicopathological parameters. $\mathrm{P}$-value $<0.05$ was considered significant.

\section{Results}

The mean age for the patients at diagnosis was 65.59 \pm 12.03 years (range, $24-98$ years). One hundred and fifty patients $(71.1 \%)$ were male and sixty-one patients $(28.9 \%)$ were female. Tumor Subtypes were Intestinal, Diffuse, Musinous, Mixed with 142(67.3\%),43(20.4\%), 16(7.6\%), $10(4.7 \%)$ values, respectively. Tumor differentiation was divided into three grades: well differentiated (low grade) with 94 patients $(44.5 \%)$, moderately differentiated (intermediate grade) with 63 patients $(29.9 \%)$ and poorly differentiated (high grade) with 54 patients $(25.6 \%)$. IHC staining of HER-2 protein is scored qualitatively as $0 / 1+/ 2+/ 3+$ with $74(35.2 \%) / 53(25.2 \%) /$ $54(25.6 \%) / 25(11.8 \%)$ values in our patients and we missed HER-2 score of $5(2.4 \%$ ) patients (Table 1 ). IHC staining

Table 2. The Correlation between Her2-neu Scores and a Number of Variables in 206 Patients

\begin{tabular}{lrlrr}
\hline Variables, $\mathrm{n}(\%)$ & Score 0 and 1 & Score 2 & Score3 & P-value* \\
\hline Sex & & & & 0.155 \\
Male & $87(60)$ & $43(29.7)$ & $15(10.3)$ & \\
Female & $40(65.6)$ & $11(18)$ & $10(16.4)$ & \\
Location of Tumor & & & & 0.021 \\
Gastric & $121(64.3)$ & $47(25)$ & $20(10.7)$ & \\
Distal esophagus & $6(33.3)$ & $7(38.9)$ & $5(27.8)$ & \\
Tumor Subtype & & & & 0.001 \\
Intestinal & $68(49.3)$ & $47(34)$ & $23(16.7)$ & \\
Diffuse & $36(83.7)$ & $5(11.6)$ & $2(4.7)$ & \\
Musinous & $14(93.3)$ & $1(6.7)$ & $0(0)$ & \\
Mixed & $9(90)$ & $1(10)$ & $0(0)$ & \\
Tumor Differentiation & & & & 0.001 \\
Well & $43(46.7)$ & $33(35.8)$ & $16(17.5)$ & \\
Moderately & $38(63.3)$ & $15(25)$ & $7(11.7)$ & \\
Poorly & $46(85.1)$ & $6(11.1)$ & $2(3.8)$ & \\
\hline * Chi-square test & & & &
\end{tabular}

Table 3. The Correlation between age Groups and A Number of Variables in 211 Patients

\begin{tabular}{lclc}
\hline Variables, n $(\%)$ & $\begin{array}{c}\text { Age }<60 \\
(\mathrm{n}=73)\end{array}$ & $\begin{array}{c}\text { Age } \geq 60 \\
(\mathrm{n}=138)\end{array}$ & P-value* \\
\hline Tumor Subtype & & & 0.447 \\
$\quad$ Intestinal & $45(31.7)$ & $97(68.3)$ & \\
Diffuse & $18(41.9)$ & $25(58.1)$ & \\
Musinous & $5(31.2)$ & $11(68.8)$ & \\
Mixed & $5(50)$ & $5(50)$ & \\
Tumor Differentiation & & & 0.032 \\
$\quad$ Well & $35(37.2)$ & $59(62.8)$ & \\
Moderately & $14(22.2)$ & $49(77.8)$ & \\
Poorly & $24(44.4)$ & $30(55.6)$ & \\
Her2-neu Score (n=206) & & & \\
Score 0 and 1 & $46(35.9)$ & $82(64.1)$ & \\
Score 2 & $16(29.6)$ & $38(70.4)$ & \\
Score 3 & $9(36)$ & $16(64)$ & \\
\hline
\end{tabular}

*Chi-square test 


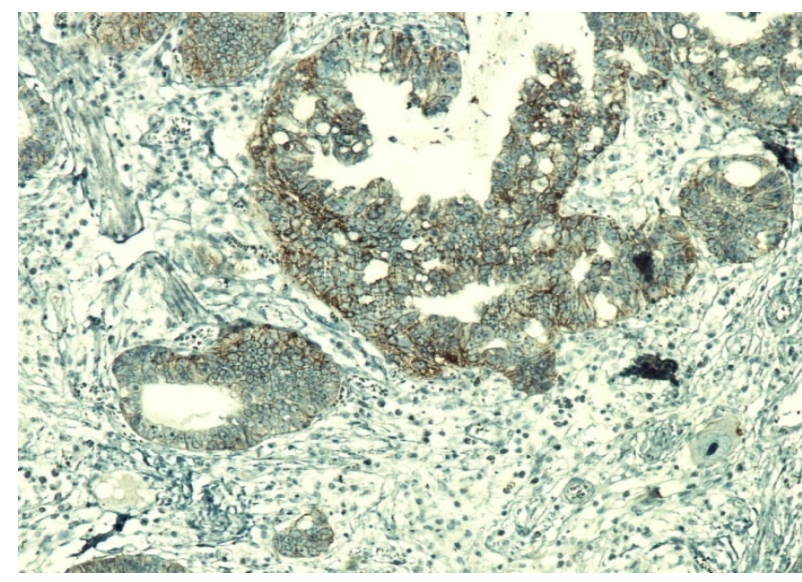

Figure 1. IHC Staining $(\times 100)$ for Her2-neu (1+) in Tumor Gastric Adenocarcinoma

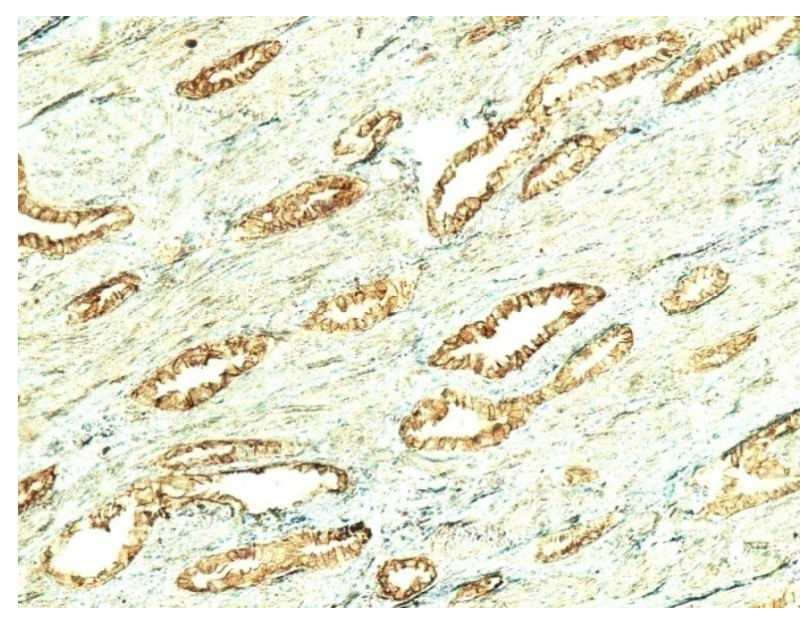

Figure 2. ?HC Staining (×100) Her2-neu (2+) in Tumor Gastric Adenocarcinoma

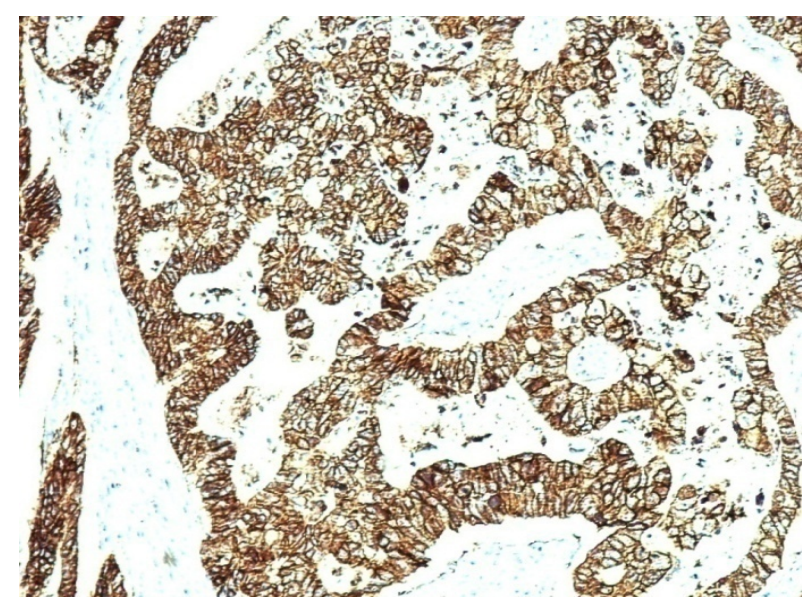

Figure 3. IHC Staining $(\times 100)$ Her2-neu (3+) in Tumor Gastric Adenocarcinoma

of HER-2 protein for score 1+, score 2+ and score $3+$ has been shown in Figures 1, 2 and 3, respectively.

The Table 2 shows the correlation between Her2-neu scores and number of variables in 206 patients ( 5 patients lost HER2-neu scores), that HER2-neu positivity rates is higher in GEJ adenocarcinomas $(\mathrm{P}<0.05)$. There is the correlation between HER2-neu scores with the intestinal type of adenocarcinoma and also with well differentiated tumor $(\mathrm{P}<0.005)$. Therefore, HER2-neu $(3+)$ is more in intestinal subtype and well differentiated tumor.

We divided the patients to two age groups $(<60$ or $\geq 60$ years). The Table 3 shows the correlation between age groups and a number of variables in 211 patients. Well differentiated tumor happens more in patients with age $<60$ years and also that this correlation was statistically significant $(\mathrm{P}<0.05)$.

\section{Discussion}

There is increasing evidence that HER2-neu is an important biomarker in GC and GEJ tumors; (Gravalos and Jimeno, 2008) analysis of HER2-neu status by IHC and in situ hybridization techniques, using different scoring methods or assays, suggests that HER2-neu is overexpressed in $7-34 \%$ of GC (Hofmann et al., 2008) and this percentage increases to $33 \%$ in GEJ tumors (Albarello et al., 2011). Also, a review study reported that Up to a fifth of gastric carcinomas and up to a third of GEJ carcinomas are positive for HER2 overexpression amplification (Hechtman and Polydorides, 2012). Almost all primary tumors with an IHC grade of 0 or $1+$ showed no amplification by FISH, whereas all IHC 3+ cases showed amplification. Of the IHC2+ cases, only about one-sixth showed HER2-neu amplification (Kim et al., 2011). Although FISH shows a high sensitivity and specificity and remains a criterion for determining gene amplification status, IHC for HER-2 protein expression may be a good alternative when FISH can't be performed (Yan et al., 2011). Therefore, reliable separation of IHC $1+/ 0$ and IHC 2+ may be difficult in biopsy samples, and FISH analysis should be used for definitive classification (Shan et al., 2013). Detecting HER2-neu status has important significance in diagnosis of GC and GEJ, (Wolff et al., 2007) and pathologists now routinely evaluate HER2neu protein expression in gastroesophageal tumors to identify patients who may benefit from the addition of trastuzumab(molecular targeted therapy) (Jeung et al., 2012). A number of studies, (Wang et al., 2011; Wu et al., 2011; Yan et al., 2011; Shan et al., 2013) reported that in gastric adenocarcinoma patients, HER2-neu (3+) rate is between $5-10 \%$, but in this study is more (10.6\%). In addition, HER2-positivity varied by tumor site, with higher rates of HER2-positivity in GEJ adenocarcinoma than in GC in this study (27.8\% vs. $10.6 \%$ respectively; $\mathrm{P}<0.05$ ), which is consistent with the results of other studies (Hofmann et al., 2008; Ruschoff et al., 2010; Kunz et al., 2012; Shan et al., 2013). A study reported 145 gastric carcinoma tissue samples, $98(67.6 \%)$ were scored as $0,25(17.2 \%)$ as $1,12(8.3 \%)$ as 2 , and $10(6.9 \%)$ as 3(Yan et al., 2011). In our study for GC and GEJ (211 cases), $74(35.1 \%)$ were scored as $0,53(25.1 \%)$ as 1,54 (25.6\%) as 2, and 25(11.8\%) as 3. Analyses demonstrated a statistically significant correlation between the expressions of HER2-neu with the pathological grade of the gastric tumors $(\mathrm{P}<0.05)$ (Tanner et al., 2005; Rakhshani et al., 2014). Our study had $17.5 \%$ poorly differentiated cancers, $11.7 \%$ moderately differentiated cancers and $3.8 \%$ well differentiated cancers in score $3+$ from HER2-neu 
overexpression and another study $46.2 \%, 30.7 \%, 23.1 \%$, respectively (Rakhshani et al., 2014). Some studies showed that HER2-neu overexpression associated with the intestinal-type carcinomas (Lauren classification) (Gravalos and Jimeno, 2008; Shan et al., 2013; Rakhshani et al., 2014). A study reported that intestinal-type carcinoma for HER-2/neu positivity prevalence rate was $16.8 \%$ in $v s .2 .3 / 8.4 \%$ in diffuse-/mixed-type cancers, (Shan et al., 2013) and also in our study, intestinal subtype was higher than other subtypes. There was no significant relation between clinicopathologic variables (sex and age of the patients, with tumor diameter, differentiation or location), (Chen et al., 2006) but we found that well differentiation tumor happened more in patients with age $<60$ years $(\mathrm{P}<0.05)$.

In patients with advanced cancer of GC and GEJ, surgery alone is not curative and other forms of therapy (molecular targeted therapy) may be required to prolong patient survival. Also, HER2-neu overexpression was more associated with intestinal cancer subtype that this can be guideline for new complementary therapy in these patients.

\section{References}

Albarello L, Pecciarini L, Doglioni C (2011). HER2 testing in gastric cancer. Adv Anat Pathol, 18, 53-9.

Chen B, Luo RC, Cui F, Qian XY(2006). Association of HER$2 /$ neu expression with prognosis of gastric cancer. Nanfang Yike Daxue Xuebao, 26, 344-47 (in Chinese).

Geddert H, Zeriouh M, Wolter M, et al (2002). Gene amplification and protein overexpression of c-erb-b2 in Barrett's carcinoma and its precursor lesions. Am J Clin Pathol, 118, 60-6.

Gravalos C, Jimeno A (2008). HER2 in gastric cancer: a new prognostic factor and a novel therapeutic target. Ann Oncol, 19, 1523-9.

Hechtman JF, Polydorides AD (2012). HER2/neu gene amplification and protein overexpression in gastric and gastroesophageal junction adenocarcinoma: a review of histopathology, diagnostic testing, and clinical implications. Arch Pathol Lab Med, 136, 691-7.

Hofmann M, Stoss O, Shi D, et al (2008). Assessment of a HER2 scoring system for gastric cancer: results from a validation study. Histopathol, 52, 797-805.

Hongo M, Nagasaki Y, Shoji T (2009). Epidemiology of esophageal cancer: orient to Occident. Effects of chronology, geography and ethnicity. J Gastroenterol Hepatol, 24, 729-35.

Jeung J, Patel R, Vila L, Wakefield D, Liu C (2012). Quantitation of HER2/neu expression in primary gastroesophageal adenocarcinomas usingconventional light microscopy and quantitative image analysis. Arch Pathol Lab Med, 136, 610-7.

Kim MA, Lee HJ, Yang HK, Bang YJ, Kim WH (2011). Heterogeneous amplification of ERBB2 in primary lesions is responsible forthe discordant ERBB2 status of primary and metastatic lesions in gastric carcinoma. Histopathol, 59, 822-31.

Kunz PL, Mojtahed A, Fisher GA, et al (2012). HER2 expression in gastric and gastroesophageal junction adenocarcinoma in a US population: clinicopathologic analysis with proposed approach to HER2 assessment. Appl Immunohistochem Mol Morphol, 20, 13-24.

Moelans CB, van Diest PJ, Milne AN, Offerhaus GJ(2011).
HER-2/neu testing and therapy in gastroesophageal adenocarcinoma. Patholog Res Int, 2011, 674182.

Rakhshani N, Kalantari E, Bakhti H, Sohrabi MR, Mehrazma M (2014). Evaluation of HER-2/neu overexpression in gastric carcinoma using a tissue microarray. Asian Pac J Cancer Prev, 15, 7597-602.

Reichelt U, Duesedau P, Tsourlakis MCh, et al (2007). Frequent homogeneous HER-2 amplification in primary and metastatic adenocarcinoma of theesophagus. Mod Pathol, 20, 120-9.

Ross JS, McKenna BJ (2001). The HER-2/neu oncogene in tumors of the gastrointestinal tract. Cancer Invest, 19, 554-68.

Ruschoff J, Dietel M, Baretton G, et al (2010). HER2 diagnostics in gastric cancer-guideline validationand development of standardized immunohistochemical testing. Virchows Arch, 457, 299-307.

Shan L, Ying J, Lu N (2013). HER2 expression and relevant clinicopathological features in gastric and gastroesophageal junction adenocarcinoma in a Chinese population. Diagn Pathol, 8, 76.

Tanner M, Hollmen M, Junttila TT, et al (2005). Amplification of HER-2 in gastric carcinoma: association with Topoisomerase II $\alpha$ gene amplification, intestinal type, poor prognosis and sensitivity to trastuzumab. Ann Oncol, 16, 273-78.

Wang YK, Gao CF, Yun T, et al (2011). Assessment of ERBB2 and EGFR gene amplification and protein expression in gastric carcinoma by immunohistochemistry and fluorescence in situ hybridization. Molecular Cytogenetics, 4, 14.

Wolff AC, Hammond MEH, Schwartz JN, et al (2007). American society of clinical oncology/college of American pathologists guideline recommendations for human epidermal growth factor receptor 2 testing in breast cancer. Arch Pathol Laboratory Med, 131, 18-43.

Wu HM, Liu YH, Lin F, et al (2011). [Association of HER2 protein expression with clinicopathologic features and prognosis in Chinese patients with gastric carcinoma]. Zhonghua Bing Li Xue Za Zhi, 40, 296-99.

Yan SY, Hu Y, Fan JG, et al (2011). Clinicopathologic significance of HER-2/neu protein expression and gene amplification in gastriccarcinoma. World J Gastroenterol, 17, 1501-6. 